\title{
Clinicopathological Features of Breast Cancer with Polysomy 17 and Its Response to Neoadjuvant Chemotherapy
}

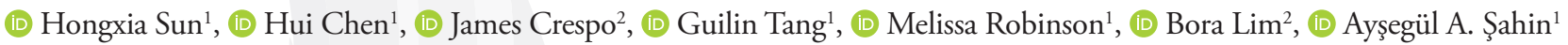

${ }^{1}$ Department of Pathology, The University of Texas MD Anderson Cancer Center, Houston, Texas

${ }^{2}$ Department of Breast Medical Oncology, The University of Texas MD Anderson Cancer Center, Houston, Texas

\begin{abstract}
Objective: The interpretation of human epidermal growth factor receptor 2 (HER2) fluorescence in situ hybridization (FISH) results may be challenging in tumors with polysomy 17 , which is defined as increased signals of chromosome enumeration probe 17 (CEP17). The effect of polysomy 17 on HER2 protein expression and tumor treatment response has not been established. In this retrospective study, we investigated the clinicopathological features of breast cancer with polysomy 17 and determined the tumors' response to neoadjuvant chemotherapy (NACT).

Materials and Methods: The study included 366 patients with primary breast cancer whose tumors had a CEP17 count of $\geq$ three/nucleus based on HER2 FISH studies. These cases were categorized according to HER2/CEP17 ratio and HER2 signals/nucleus using the American Society of Clinical Oncology/College of American Pathologists (ASCO/CAP) guidelines. We compared the clinicopathological characteristics and tumor response to NACT among different groups.

Results: There was a statistically significant difference in patients' age at diagnosis, tumor pathological grade, estrogen and progesterone receptor status, and NACT response among different HER2 FISH groups. Polysomy 17 tumors in group 1 had a higher rate of response (pathological complete response and residual cancer burden class I) to NACT containing anti-HER2 reagent than did those in other groups $(\mathrm{p}=0.004)$, whereas polysomy 17 tumors in group 3 did not show a significant response to anti-HER2 treatment.
\end{abstract}

Conclusion: Polysomy 17 tumors in different HER2 FISH groups have different pathological features and respond to NACT differently. These results may help us identify patients who will benefit from anti-HER2 therapy.

Keywords: Polysomy 17, breast cancer, HER2 FISH study, neoadjuvant chemotherapy

Cite this article as: Sun H, Chen H, Crespo J, Tang G, Robinson M, Lim B, Şahin AA. Clinicopathological Features of Breast Cancer with Polysomy 17 and Its Response to Neoadjuvant Chemotherapy. Eur J Breast Health 2021; 17(2): 128-136

\section{Key Points}

- Polysomy 17 tumors show different clinicopathological characteristics among different HER2 FISH groups.

- Polysomy 17 tumors show a different response to NACT among different HER2 FISH groups.

- Polysomy 17 tumors in group 3 did not show a significant response to anti-HER2 treatment.

\section{Introduction}

Human epidermal growth factor receptor 2 (HER2) is a member of the human epidermal growth factor receptor family, encoded by the gene HER2 on 17q12-21.32 (1). Around 20\% of invasive breast cancer cases overexpress or exhibit amplification of HER2 (HER2+ breast cancer). HER2+ breast cancer is known to be an aggressive disease, with a poor clinical outcome (2). Trastuzumab, a monoclonal antibody that targets HER2, has demonstrated efficacy against HER2+ primary and metastatic breast cancer, both as a single agent and combined with chemotherapy $(3,4)$. Treatments that include anti-HER2 reagent have become the standard of care for patients with early or advanced HER2+ breast cancer $(5,6)$.

Accurate detection of HER2 overexpression or gene amplification is crucial in determining patients' eligibility for anti-HER2 treatment and predicting disease prognosis. According to the American Society of Clinical Oncology/College of American Pathologists (ASCO/CAP) guidelines, HER2 testing is performed using immunohistochemical (IHC) assessment of HER2 protein overexpression and in situ hybridization 
(ISH) analysis of HER2 gene amplification (7). ISH is conducted using either a single probe to enumerate HER2 copies per nucleus only or a dual-probe technique in which the HER2/CEP17 ratio is determined via hybridization to the chromosome 17 centromere region using chromosome enumeration probe 17 (CEP17). Although ASCO/CAP provides clear guidance on HER2 assessment, the test results can be difficult to interpret for various reasons, including copy number alterations in different foci on chromosome 17.

Chromosome 17 polysomy is associated with equivocal HER2 results. True polysomy is defined as the presence of extra copies of one or a whole chromosome. However, according to recent studies, true chromosome 17 polysomy is very rare in breast cancer. Focal amplifications encompassing the centromere are a common cause of the increase in CEP17 signals in ISH testing (8). Currently, the commonly adopted threshold for polysomy 17 is a mean of $\geq 3$ CEP17 signals per nucleus (9). Increased CEP17 copies can alter the HER2/ CEP17 ratio and subsequently influence the interpretation of the final HER2 ISH result. Consequently, 2013 ASCO/CAP recommends the use of a reflex test with alternative chromosome 17 probes for resolving equivocal HER2 ISH results (7).

HER2 FISH testing using alternative chromosome 17 probes can be performed by testing for additional genes on chromosome 17 that are not expected to coamplify with HER2. The commonly used commercially available probes include SMS (Smith-Magenis syndrome, also called $R A I 1), R A R A$ (retinoic acid receptor alpha), and TP53 (8). On using these different chromosome 17 genes, HER2 gene status has been reported to be upgraded from equivocal to amplified or positive in a significant percentage of cases $(8,10$, 11). However, in these studies, there were no clinical outcome data available in these patients with "revised" HER2+ breast cancer. The benefit of HER2-targeted therapy in these patient populations was also unknown. A recent study by Sneige et al. (12) demonstrated that the "revised" HER2 status due to the use of alternative chromosome 17 probes was unreflective of patient outcome. They concluded that these alternative chromosome 17 genes might overestimate the number of HER2-positive cases and lead to an erroneous upgrade of HER2 status to "positive."

A better understanding of the biological features of polysomy 17 breast cancer and how polysomy 17 affects HER2 gene copy number and protein expression could help select patients who will respond to antiHER2 treatment. In this study, we determined the clinicopathological characteristics of patients with breast cancer with polysomy 17 . We also investigated the tumors' response to neoadjuvant chemotherapy (NACT), with and without anti-HER2 reagent.

\section{Materials and Methods}

\section{Patient cohort}

This retrospective study was conducted in a cohort of 366 patients with primary invasive breast cancer. Tumor HER2 FISH testing was performed at our institution between April $1^{\text {st }}, 2013$, and March $31^{\text {st }}$, 2018 , and a CEP count $\geq 3 /$ nucleus was required. Patients with a prior history of breast cancer, those diagnosed with de novo stage IV disease, and those who had multiple HER2 FISH tests using the same specimen were excluded from the study.

We reviewed patients' medical charts to determine clinical variables, including age at diagnosis, tumor pathological characteristics (tumor size, lymph node status, pathological staging, and histological grade), tumor biomarker features [estrogen receptor (ER), progesterone receptor (PR), Ki-67 value, and HER2 immunohistochemical (IHC) analysis], and treatment (with or without NACT and with or without anti-HER2 reagent in the NACT regimen). In patients who received NACT, tumor response to NACT was evaluated according to the pathological residual cancer burden (RCB) (13). A pathological complete response (pCR) and RCB class I were interpreted as a good response, while RCB classes II and III were interpreted as a poor response. Approval was obtained from the Institutional Review Board at our institution (no: PA18-0021) before the initiation of this study.

\section{Immunohistochemical analysis}

The IHC analysis performed at our department was processed using formalin-fixed, paraffin-embedded tumor sections $(4 \mu \mathrm{m})$ with ER Clone 6F11 (Leica Biosystems, Inc., Buffalo Grove, IL) and PR Clone PgR 1294 (Agilent DAKO, Santa Clara, CA). The HER2 IHC analysis was performed using antibody clone AB8 (NeoMarkers) from April $1^{\text {st }}, 2013$ until August 31 $1^{\text {st }}, 2016$, and clone 4B5 (Ventana Medical Systems, Inc., Tucson, AZ) from September $1^{\text {st }}, 2016$, to March $31^{\text {st }}$, 2018, due to institutional antibody change. The IHC studies (ER, $\mathrm{PR}$, and HER2) conducted at outside institutions were reviewed at our department. ER, PR, and HER2 IHC statuses were interpreted according to the ASCO/CAP guidelines $(7,14)$.

\section{FISH analysis}

HER2 FISH analysis was performed using the Vysis PathVysion probe kit, which includes a SpectrumGreen-conjugated probe for the alpha satellite DNA located at the centromeric region of chromosome 17 (17p11.1-q11.1) and SpectrumOrangeconjugated probe for the HER2 gene locus (Abbott Molecular/ Abbott Laboratories, Abbott Park, IL). The same specimen blocks used for the HER2 IHC study were selected for the FISH study. HER2 and CEP17 signals in 60 representative invasive cell nuclei were examined. HER2 FISH result was interpreted according to the ASCO/CAP guidelines (7). For cases that were interpreted as equivocal for HER2 amplification, another 60 representative invasive cell nuclei were examined.

Polysomy 17 tumors are classified into four HER2 FISH groups according to the HER2/CEP17 ratio and HER2 signals/nucleus, on the basis of ASCO/CAP guidelines: group 1 had a HER2/CEP17 ratio $\geq 2.0$ and $H E R 2$ signals/nucleus $\geq 4$.0; group 3 had a HER2/ CEP17 ratio $<2.0$ and HER2 signals/nucleus $\geq 6.0$; group 4 had a HER2/CEP17 ratio $<2.0$ and HER2 signals/nucleus $\geq 4.0$ and $<6.0$; group 5 had a HER2/CEP17 ratio $<2.0$ and HER2 signals/nucleus $<4.0$ (15).

\section{Statistical analysis}

Categorical variables were summarized using frequencies and percentages. For most categorical clinical and pathological features, group differences were assessed using chi-square and Fisher's exact test. The correlation between the age at diagnosis and tumor HER2 FISH group was examined by ANOVA analysis. A p-value $<0.05$ (two-sided) was considered statistically significant.

\section{Results}

\section{Group distribution of polysomy 17 tumors}

Among the 366 primary invasive breast cancer tumors included in this study, $128(35.0 \%)$ were classified as HER2 FISH group 1, 21 
(5.7\%) as group 3, $69(18.9 \%)$ as group 4, and $148(40.4 \%)$ as group 5. Representative HER2 FISH images of polysomy 17 tumors in each group are shown (Figure 1).

\section{Clinicopathological characteristics of polysomy 17 tumors}

The clinicopathological characteristics of invasive breast cancer with polysomy 17 are shown in Table 1 . In our study cohort, the mean age of patients at diagnosis was 56 years (range: 24-92 years). Most tumors were of a ductal type [340 of 366 (92.9\%)] and histological grade 2 [151 of $365(41.4 \%)$ ] or [201 of $365(55.1 \%)]$ with ER expression in over two-thirds of the patients [260 of $366(71.0 \%)$ ] and PR expression in half of the patients [184 of 365 (50.4\%)].

We investigated the clinicopathological characteristics of polysomy 17 tumors in 4 HER2 FISH groups. The histological types did not differ significantly; however, there were statistically significant differences in the age of the patients at diagnosis, tumor nuclear and histological grade, and ER/PR status. Patients with group 1 polysomy 17 tumors were diagnosed at a slightly younger age (mean age: 54 years) than were those with groups 4 (mean age: 57 years) and 5 (mean age: 58 years) tumors $(\mathrm{p}<0.05)$. Histological grade 3 tumors were more frequently observed in group 1 [ 81 of $127(63.8 \%)$ ] than in groups 3 [6 of 21 $(28.6 \%)]$ and 5 [73 of $148(49.3 \%)](\mathrm{p}<0.05)$. ER negative tumors were more common in group 1 [52 of $128(40.6 \%)$ ] than in groups 4 [14 of $69(20.3 \%)]$ and 5 [35 of $148(23.6 \%)](\mathrm{p}<0.05)$. PR negative tumors were more common in group 1 [86 of $128(67.2 \%)$ ] than in the other three groups [5 of $21(23.8 \%)$ in group 3; 26 of $69(37.7 \%)$ in group 4; 64 of 147 (43.5\%) in group 5] $(\mathrm{p}<0.001)$.

Next, we evaluated tumor size and axillary lymph node status in 185 polysomy 17 tumors without NACT treatment (Table 2). These tumors were predominantly low stage [109 (58.9\%) were pT1 and 63 (34.1\%) were p T2], and only $13(7 \%)$ were pT3 or pT4. No differences were observed in tumor pathological staging among the four groups. One-third of the tumors [52 of $185(30.2 \%)]$ had metastasized to the axillary lymph nodes at the time of surgery. Tumors in group 1 had a significantly lower metastatic rate than did tumors in group $5(16.7 \%$ vs $37.0 \%, \mathrm{p}<0.05)$.

\section{Comparison of IHC and FISH for HER2 status in polysomy 17 tumors}

The HER2 IHC and FISH test results of the 366 tumors are outlined in Table 3: 92 tumors (26.3\%) were positive (score 3+) on HER2 IHC

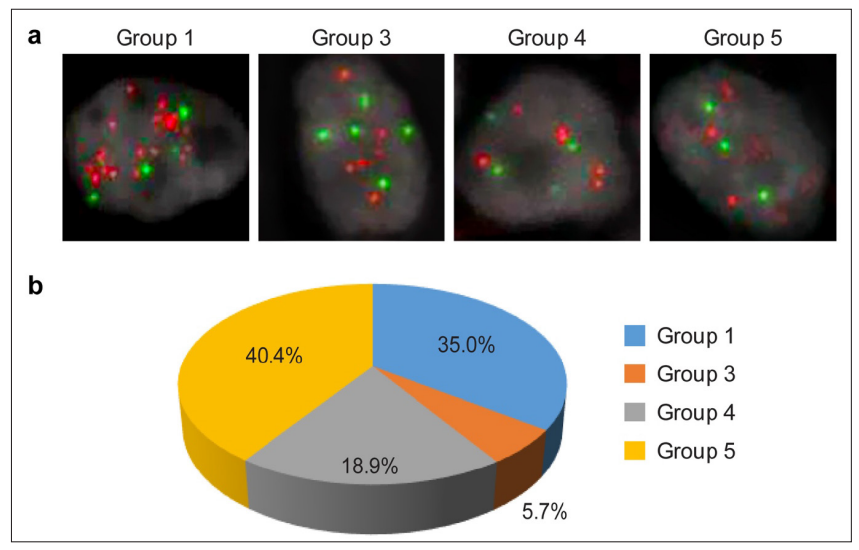

Figure 1. Representative HER2 FISH images of polysomy 17 tumors in different HER2 FISH groups (a); proportions of each group (b)

HER2: Human epidermal growth factor receptor 2; FISH: Fluorescence in situ testing, 145 (41.4\%) were equivocal (score $2+)$, and $113(32.3 \%)$ were negative, with a score of $1+$ [92 of $113(81.4 \%)$ ] or 0 [21 of 113 (18.6)].

The distribution of HER2 IHC results was significantly different among tumors in the four HER2 FISH groups ( $\mathrm{p}<0.001)$. In group 1 , most tumors [86 of $123(69.9 \%)$ ] were positive (score $3+$ ) on HER2 IHC testing, about a quarter [34 of $123(27.6 \%)$ ] were equivocal (score $2+$ ), and only a small number [3 of $123(2.5 \%)$ ] were negative (score $1+$ ). No tumors had a HER2 IHC score of 0 in this group.

In contrast, most tumors [75 of $138(54.4 \%)]$ in group 5 were negative for HER2 IHC staining, with a score of $1+$ [60 of $138(43.5 \%)]$ or 0 [15 of $138(10.9 \%)$ ]. Fewer tumors in this group [62 of $138(44.9 \%)$ ] were equivocal (score $2+$ ), and only one tumor $(0.7 \%)$ was positive (score 3+). In group 3, five tumors $(23.8 \%$ ) were positive (score 3+) on HER2 IHC testing; this was significantly lower than the number of tumors in group $1(\mathrm{p}<0.001)$ but higher than that in groups 4 $(\mathrm{p}<0.05)$ and $5(\mathrm{p}<0.001)$.

\section{NACT response in polysomy 17 tumors}

In our study cohort, 181 patients underwent NACT after initial diagnosis. Of these, 97 patients received an anti-HER2 therapy, and 84 did not. RCB was calculated for 175 tumors to evaluate the response to NACT (13). RCB could not be calculated for six tumors because of insufficient parameters of residual cancer in the breast or lymph nodes after NACT (all cases were from outside facilities). As shown in Table 4, 82 of 175 patients (46.9\%) experienced a good response, while 93 $(53.1 \%)$ had a poor response to NACT.

Tumors in different HER2 FISH groups had different responses to NACT $(\mathrm{p}<0.001)$. Overall, a significant number group 1 tumors had a good response compared with tumors in other groups: 56 tumors (70.9\%) in group 1 had a pCR or RCB I compared with three tumors $(30.0 \%)$ in group $3(\mathrm{p}<0.05)$, eight $(30.8 \%)$ in group $4(\mathrm{p}<0.001)$, and $15(25 \%)$ in group $5(\mathrm{p}<0.001)$. Most patients with group 1 or 3 tumors received an anti-HER2 reagent containing NACT. Of these, 55 tumors $(72.4 \%)$ in group 1 had a good response compared with three of nine in group $3(33.3 \%)(\mathrm{p}<0.004)$. In contrast, most patients with group 4 or 5 tumors did not receive an anti-HER2 reagent; $33.3 \%$ and $26.8 \%$ of these tumors had a good response, respectively.

We also investigated the pathological features associated with tumor response to NACT, with or without anti-HER2 therapy. HER2 FISH group 1, ER negativity, PR negativity, and HER2 overexpression were associated with a good response to treatment with anti-HER2 reagent (Table 5). A high nuclear and histological grade, ER negativity, PR negativity, and high proliferative index Ki67 were associated with a good response to treatment without antiHER2 reagent (Table 6).

\section{Discussion and Conclusion}

Polysomy has been proposed to explain the increased rates of HER2 amplification or discordance between IHC and FISH results. The results of recent studies have suggested that, detected by FISH, the major contributor to polysomy 17 is a significantly increased copy number of CEP17 secondary to the amplification of larger segments of chromosome 17, involving both HER2 and the centromere (8). An elevated CEP17 count is frequently observed in invasive breast cancer. Using the cut-off of $\geq 3$ CEP17 copies per cell, reported prevalence 
rates of polysomy 17 tumors have ranged from 3\% to $46 \%$ across various studies (16-23).

Polysomy 17 contributes to increased HER2 FISH equivocal results. To resolve this problem, the 2013 ASCO/CAP guidelines advocate additional testing in these cases. Many studies have tested for additional genes on chromosome 17 and have upgraded a significant portion of equivocal cases to HER2 amplified or positive (10-12). However, recent studies have demonstrated the presence of frequent complex structural alterations of chromosome 17 in patients with breast cancer, with losses and gains of genetic material at different loci of the chromosome $(10,24)$. As a consequence, the use of additional
FISH probes is not sufficient for correcting the HER2 gene status. Currently, there is no standard method to detect the HER2 gene status in polysomy 17 breast cancer.

The reported clinicopathological features of polysomy 17 tumors are controversial. Several studies have linked polysomy with unfavorable pathological features, such as high proliferative activity, high Nottingham Prognostic Index Score, and nodal involvement (19, 23, 25), while other studies have found no significant differences between polysomic and nonpolysomic primary breast cancer in terms of clinicopathological variables and patient survival $(18,21)$. In our study cohort, most of the polysomy 17 tumors are histological grade

Table 1. Clinical and pathological characteristics of patients with primary breast cancer with polysomy 17 tumors

\begin{tabular}{|c|c|c|c|c|c|c|}
\hline Characteristic & $\begin{array}{c}\text { Total } \\
(\mathrm{n}=366)\end{array}$ & $\begin{array}{l}\text { Group } 1 \\
(n=128)\end{array}$ & $\begin{array}{l}\text { Group } 3 \\
(n=21)\end{array}$ & $\begin{array}{l}\text { Group } 4 \\
(n=69)\end{array}$ & $\begin{array}{l}\text { Group } 5 \\
(n=148)\end{array}$ & p-value \\
\hline \multicolumn{7}{|l|}{ Age, years } \\
\hline Mean & 56 & 54 & 55 & 57 & 58 & \\
\hline \multirow[t]{2}{*}{ Range } & 24-92 & 24-90 & $34-75$ & $32-92$ & $24-85$ & $0.044^{+}$ \\
\hline & & & & * & * & \\
\hline \multicolumn{7}{|c|}{ Histological type, n (\%) } \\
\hline IDC, NOS & 340 (92.9) & $122(95.3)$ & $20(95.2)$ & $65(94.2)$ & $133(89.9)$ & \\
\hline ILC & $9(2.5)$ & $2(1.6)$ & $1(4.8)$ & $1(1.4)$ & $5(3.4)$ & $0.701^{++}$ \\
\hline Other & $17(4.6)$ & $4(3.1)$ & $0(0)$ & $3(4.4)$ & $10(6.7)$ & \\
\hline \multicolumn{7}{|c|}{ Nuclear grade, n (\%) } \\
\hline I & $2(0.5)$ & $1(0.7)$ & $0(0)$ & $0(0)$ & $1(0.7)$ & \\
\hline II & $151(41.3)$ & $39(30.5)$ & 15 (71.4) & $27(39.1)$ & $70(47.3)$ & $0.004^{++}$ \\
\hline \multirow[t]{2}{*}{ III } & $213(58.2)$ & $88(68.8)$ & $6(28.6)$ & $42(60.9)$ & $77(52)$ & 0.004 \\
\hline & & & $* *$ & & * & \\
\hline \multicolumn{7}{|c|}{ Histological grade, n (\%) } \\
\hline 1 & $13(3.5)$ & $2(1.6)$ & $1(4.7)$ & $3(4.4)$ & $7(4.8)$ & \\
\hline 2 & $151(41.4)$ & $44(34.6)$ & $14(66.7)$ & $25(36.2)$ & $68(45.9)$ & \\
\hline 3 & $201(55.1)$ & $81(63.8)$ & $6(28.6)$ & $41(59.4)$ & $73(49.3)$ & $0.04^{++}$ \\
\hline \multirow[t]{2}{*}{ NA } & 1 & 1 & 0 & 0 & 0 & \\
\hline & & & * & & * & \\
\hline \multicolumn{7}{|l|}{ ER, n (\%) } \\
\hline Positive & $260(71.0)$ & $76(59.4)$ & $16(76.2)$ & 55 (79.7) & $113(76.4)$ & \\
\hline \multirow[t]{2}{*}{ Negative } & $106(29.0)$ & $52(40.6)$ & $5(23.8)$ & $14(20.3)$ & 35 (23.6) & $0.005^{++}$ \\
\hline & & & & * & * & \\
\hline \multicolumn{7}{|l|}{ PR, n (\%) } \\
\hline Positive & $184(50.4)$ & $42(32.8)$ & $16(76.2)$ & $43(62.3)$ & $83(56.5)$ & \\
\hline Negative & $181(49.6)$ & $86(67.2)$ & $5(23.8)$ & $26(37.7)$ & $64(43.5)$ & $<0.001^{++}$ \\
\hline \multirow[t]{2}{*}{ NA } & 1 & 0 & 0 & 0 & 1 & - \\
\hline & & & $* *$ & $* *$ & $* *$ & \\
\hline
\end{tabular}

Important p-values shown in bold.

${ }^{*} \mathrm{p}<0.05$ compared with group $1 ;$ ** $\mathrm{p}<0.001$ compared with group 1 ; +: one-way ANOVA analysis; ${ }^{+*}$ : Chi-square analysis; IDC: Invasive ductal carcinoma; NOS: Invasive ductal carcinoma of no specific type; ILC: Invasive lobular carcinoma; ER: Estrogen receptor; PR: Progesterone receptor; NA: Not available; n: Number 
2 or 3 . ER positivity was seen in $71.4 \%$ of tumors, which is a slightly lower rate than the known $75 \%-80 \%$ rate in invasive breast cancer (Table 1). This indicates that polysomy 17 tumors display unfavorable pathological features.

In addition, polysomic HER2-amplified tumors have been reported to have more unfavorable pathological features than polysomic HER2-nonamplified tumors (20). Since HER2 FISH group 1 tumors are HER2 amplified and HER2 FISH group 5 tumors are HER2 nonamplified, we compared the clinicopathological features of the tumors in these two groups. Group 1 tumors were more likely to be diagnosed in younger patients $(\mathrm{p}<0.01)$, have a higher histological grade $(\mathrm{p}<0.05)$, and be ER negative $(\mathrm{p}<0.01)$ and $P R$ negative $(\mathrm{p}<0.001)$ than were group 5 tumors (Table 1$)$. These findings agree with previous findings that HER2-positive breast cancer is more likely to be diagnosed in younger patients and be more aggressive (2). However, when the tumor stage and axillary lymph node status in these two groups were compared, group 1 tumors were found to have a lower lymph node metastatic rate $(\mathrm{p}<0.05)$. No significant difference was observed in tumor stage between these two groups (Table 2). The reason why polysomic
HER2-amplified tumors had a lower risk of lymph node metastasis needs to be further studied.

The effect of polysomy 17 on HER2 alteration also needs to be further investigated. Some studies have shown that polysomy 17 alone might not significantly contribute to the variation in HER2 copy number and HER2 protein overexpression (16), while other studies have correlated polysomy 17 with an increased HER2 IHC score in tumors without HER2 amplification (22, 26-28). For example, Hyun et al. (27) reported a significantly higher incidence of elevated CEP17 count in tumors with HER2 IHC score 2+/3+ compared with tumors with score $0 / 1+$. In addition, Varshney et al. (22) and Petroni et al. (23) found that high CEP17 counts were associated with HER2 IHC score $3+$ staining. Our results showed that $67.7 \%$ of polysomy 17 tumors were HER2 IHC score $2+/ 3+$, and $32.3 \%$ of tumors were score $0 / 1+$. In group 5, 44.9\% of tumors were HER2 IHC score 2+, and one tumor $(0.7 \%)$ was score $3+$ (Table 3$)$. This percentage was dramatically higher than that reported by the BCIRG clinical trial, in which $0.55 \%$ of group 5 tumors (including polysomy 17 and nonpolysomy 17 tumors) had a HER2 IHC score $2+/ 3+(14)$. Although this difference could be due to variations in the population under study

Table 2. Pathological stage of polysomy 17 tumors that had not received NACT

\begin{tabular}{|c|c|c|c|c|c|c|}
\hline & Total & Group 1 & Group 3 & Group 4 & Group 5 & \\
\hline Stage & $(n=185)$ & $(n=46)$ & $(n=11)$ & $(n=42)$ & $(n=86)$ & p-value \\
\hline \multicolumn{7}{|c|}{ Tumor stage, n (\%) } \\
\hline pT1 & $109(58.9)$ & $30(65.2)$ & $6(54.5)$ & $24(57.1)$ & $49(57)$ & \multirow{3}{*}{0.665} \\
\hline pT2 & $63(34.1)$ & $15(32.6)$ & $4(36.4)$ & $16(38.1)$ & $28(32.6)$ & \\
\hline $\mathrm{pT} 3+\mathrm{T} 4$ & $13(7.0)$ & $1(2.2)$ & $1(9.1)$ & $2(4.8)$ & $9(10.4)$ & \\
\hline \multicolumn{7}{|c|}{ Lymph node stage, n (\%) } \\
\hline pNO & $120(69.8)$ & $35(83.3)$ & 7 (77.8) & $27(67.5)$ & $51(63)$ & \multirow{4}{*}{0.121} \\
\hline $\mathrm{pN} 1+\mathrm{N} 2+\mathrm{N} 3$ & $52(30.2)$ & $7(16.7)$ & $2(22.2)$ & $13(32.5)$ & $30(37.0)$ & \\
\hline \multirow[t]{2}{*}{ NA } & 13 & 4 & 2 & 2 & 5 & \\
\hline & & & & & * & \\
\hline
\end{tabular}

Table 3. HER2 protein expression in polysomy 17 tumors

\begin{tabular}{|c|c|c|c|c|c|c|}
\hline & Total & Group 1 & Group 3 & Group 4 & Group 5 & \\
\hline & $(n=366)$ & $(n=128)$ & $(n=21)$ & $(n=69)$ & $(n=148)$ & p-value \\
\hline \multicolumn{7}{|c|}{ HER2 IHC score, n (\%) } \\
\hline 0 & $21(6.0)$ & $0(0)$ & $2(9.6)$ & $4(5.8)$ & $15(10.9)$ & \multirow{6}{*}{$<0.001$} \\
\hline $1+$ & $92(26.3)$ & $3(2.5)$ & $7(33.3)$ & $22(32.4)$ & $60(43.5)$ & \\
\hline $2+$ & 145 (41.4) & $34(27.6)$ & $7(33.3)$ & $42(61.8)$ & $62(44.9)$ & \\
\hline $3+$ & $92(26.3)$ & $86(69.9)$ & $5(23.8)$ & $0(0)$ & $1(0.7)$ & \\
\hline \multirow[t]{2}{*}{ NA } & 16 & 5 & 0 & 1 & 10 & \\
\hline & & & $* *$ & $* *$ & $* *$ & \\
\hline
\end{tabular}


Table 4. Treatment response of polysomy 17 tumors in HER2 FISH group to NACT

\begin{tabular}{|c|c|c|c|c|c|c|}
\hline & Total & Group 1 & Group 3 & Group 4 & Group 5 & \\
\hline Treatment & $(n=181)$ & $(n=82)$ & $(n=10)$ & $(n=27)$ & $(n=62)$ & p-value \\
\hline \multicolumn{7}{|c|}{ NACT overall, n (\%) } \\
\hline $\mathrm{pCR}+\mathrm{RCB} I$ & $82(46.9)$ & $56(70.9)$ & $3(30)$ & $8(30.8)$ & $15(25)$ & \multirow{4}{*}{$<0.001$} \\
\hline RCB II + III & $93(53.1)$ & $23(29.1)$ & $7(70)$ & $18(69.2)$ & $45(75)$ & \\
\hline \multirow[t]{2}{*}{ NA } & 6 & 3 & 0 & 1 & 2 & \\
\hline & & & * & ** & ** & \\
\hline \multicolumn{7}{|c|}{ NACT with anti-HER2, n (\%) } \\
\hline $\mathrm{pCR}+\mathrm{RCB} I$ & $59(62.8)$ & $55(72.4)$ & $3(33.3)$ & $1(20)$ & $0(0)$ & \multirow{4}{*}{0.004} \\
\hline RCB II + III & $35(37.2)$ & $21(27.6)$ & $6(66.7)$ & $4(80)$ & $4(100)$ & \\
\hline \multirow[t]{2}{*}{ NA } & 3 & 2 & 0 & 1 & 0 & \\
\hline & & & * & * & * & \\
\hline \multicolumn{7}{|c|}{ NACT without anti-HER2, n (\%) } \\
\hline $\mathrm{pCR}+\mathrm{RCB} I$ & $23(28.4)$ & $1(33.3)$ & $0(0)$ & 7 (33.3) & $15(26.8)$ & \multirow{3}{*}{0.856} \\
\hline RCB II + III & 58 (71.6) & $2(66.7)$ & $1(100)$ & $14(66.7)$ & $41(73.2)$ & \\
\hline NA & 3 & 1 & 0 & 0 & 2 & \\
\hline
\end{tabular}

and test methods, the high percentage of tumors with HER2 IHC score $2+/ 3+$ in our patients with HER2-nonamplified tumors indicates that polysomy 17 is associated with an increased HER2 IHC score.

The potential association between polysomy 17 and HER 2 expression raises the question of whether polysomy 17 influences anti-HER2 treatment response. Some data indicate that polysomy 17 tumors are sensitive to anti-HER2 treatment. In a study by Hofmann et al. (17), two patients with HER2 overexpression (IHC 3+) due to polysomy rather than HER2 amplification experienced a response to trastuzumab. In contrast, phase III EGF30001 trial revealed that lapatinib had no significant benefit in patients with HER2nonamplifed, polysomic metastatic breast cancer (29). We evaluated the response of polysomy 17 tumors to NACT in the presence or absence of anti-HER2 reagent. Our results indicated that patients with HER2 FISH group 1 tumors who received NACT containing an anti-HER2 reagent had a higher good response rate than did patients with other groups of tumors who received NACT, with or without anti-HER2 reagent. In group 5 tumors, one patient had a tumor that showed HER2 overexpression (IHC score 3+). However, this patient did not undergo NACT. In reviewing the pathological features associated with tumor response to NACT, our results revealed that, for tumors treated with NACT containing an anti-HER2 reagent, the HER2 FISH group, ER and PR status, and HER2 expression level were associated with treatment response. For tumors treated without anti-HER2 reagent, tumor nuclear and histological grade, proliferative index Ki-67, and ER and PR status were correlated with response.

Another finding was that, in our study cohort, HER2 FISH group 3 tumors, which were designated as HER2-amplified tumors according to 2013 ASCO/CAP guidelines, did not demonstrate a significant response to NACT containing anti-HER2 reagent. This finding supports the recently published update to the ASCO/CAP guidelines that HER2 status in group 3 tumors should be interpreted combined with the FISH result and HER2 protein expression level (30). To our knowledge, this is the first report of the treatment response of polysomy 17 tumors in the NACT setting. Further study in a larger population is needed to confirm these findings.

In summary, we studied the clinicopathological features and tumor response to NACT treatment of polysomy 17 breast cancer on the basis of tumor HER2 FISH groups. We conclusively demonstrated that group 1 polysomy 17 tumors have more unfavorable pathological features but have the best response to NACT with anti-HER2 treatment. Polysomy 17 tumors in other groups did not significantly benefit from anti-HER2 treatment in the NACT setting. These results could help identify patients who may benefit from a more intensive targeted therapy regimen. 
Table 5. Pathological features associated with response to NACT with anti-HER2 reagent in polysomy 17 tumors

\section{PCR + RCB I RCB II + RCB III}

Feature $\quad(n=59) \quad(n=35) \quad$ p-value

HER2 FISH group, n (\%)

4

5

HER2 IHC score, n (\%)

0
$1+$
$2+$
$3+$
NA

NA

ER, n (\%)

Positive

Negative

PR, n (\%)

Positive

Negative

Ki-67, n (\%)

$<15$

$15-<35$

$\geq 35$

NA

$55(93.2)$
$3(5.1)$
$1(1.7)$
$0(0)$

$21(60.0)$

6 (17.1)

4 (11.4)

4 (11.4)

0.02
0.02
0.008

0.008

$0(0)$

2 (5.7)

1 (1.8)

$6(17.1)$

15 (26.8)

10 (28.6)

$<0.05$

40 (71.4)

17 (48.6)

3

0

-
0.02
0.02
0.008

(n)

2

3

26 (74.3)

$\begin{array}{ll}28(47.5) & 26(74.3) \\ 31(52.5) & 9(25.7)\end{array}$

$<0.05$

19 (54.3)

$\begin{array}{ll}13(22.0) & 19(54.3) \\ 46(78.0) & 16(45.7)\end{array}$

$<0.05$

$5(9.8) \quad 1(4.5)$

15 (29.4)

7 (31.8)

$31(60.8)$

14 (63.6)

0.821

8

13

Nuclear grade, n (\%)

II

III

$\begin{array}{ll}18(30.5) & 15(42.9) \\ 41(69.5) & 20(57.1)\end{array}$

0.225

\section{Histological grade, n (\%)}

$\begin{array}{cccc}1 & 1(1.7) & 0(0) & \\ 2 & 19(32.2) & 19(54.3) & 0.063 \\ 3 & 39(66.1) & 16(45.7) & \end{array}$

Important p-values are shown in bold.

NACT: Neoadjuvant chemotherapy; HER2: Human epidermal growth factor receptor 2; FISH: Fluorescence in situ hybridization; IHC: Immunohistochemical; RCB: Residual cancer burden; NA: Not available. ER: estrogen receptor; PR: progesterone receptor; n: Number

\section{ER, n (\%)}

Positive

Negative

PR, n (\%)

Positive

Negative

Her2 IHC score, n (\%)

0

$1+$

Table 6. Pathological features associated with response to NACT without anti-HER2 reagent in polysomy 17 tumors

$\mathrm{PCR}+\mathrm{RCB} \mathbf{\mathrm { R }} \quad \mathrm{RB}$ II + RCB

III

$\begin{array}{lccr}\text { Feature } & (\mathbf{n}=\mathbf{2 3}) & \mathbf{( n = 5 8 )} & \text { P-value } \\ \text { Nuclear grade, } \mathbf{n ( \% )} & & & \\ \text { II } & 2(8.7) & 28(48.3) & <0.001 \\ \text { III } & 21(91.3) & 30(51.7) & \end{array}$

Histological grade, n (\%)

(

2

$0(0)$

$1(1.7)$

$2(8.7) \quad 29(50.0)$

$<0.001$

$21(91.3) \quad 28(48.3)$

(2)

$$
2+
$$

8 (34.8)

39 (67.2)

19 (32.8)

$<0.05$

\section{NA}

HER2 FISH group, n (\%)

1

3

4

5

15 (65.2)

$27(46.6)$

$\begin{array}{ll}3(13.0) & 27(46.6) \\ 20(87) & 31(53.4)\end{array}$

$<0.05$

Ki-67, n (\%)

$<15$

4 (19) $\quad 5$ (9.4)

9 (42.9) 25 (47.2)

8 (38.1)

23 (43.4)

0.521

2

5

$\begin{array}{ll}1(4.3) & 2(3.4) \\ 0(0) & 1(1.7) \\ 7(30.4) & 14(24.1) \\ 5(65.2) & 41(70.7)\end{array}$

0.837

$$
\begin{aligned}
& 15-<35 \\
& \geq 35
\end{aligned}
$$$$
0(0)
$$$$
8 \text { (15.4) }
$$$$
2 \text { (11.8) }
$$$$
24(46.2)
$$$$
15 \text { (88.2) }
$$$$
20(38.5)
$$

$<0.05$

NA

6

6

Important $\mathrm{p}$-values are shown in bold.

NACT: Neoadjuvant chemotherapy; HER2: Human epidermal growth factor receptor 2; FISH: Fluorescence in situ hybridization; IHC: Immunohistochemical; RCB: Residual cancer burden; NA: Not available. ER: estrogen receptor; PR: progesterone receptor; n: Number 
Ethics Committee Approval: Approval was obtained from the Institutional Review Board at our institution (PA18-0021) before the initiation of this study.

Informed Consent: Retrospective study.

Peer-review: Externally peer-reviewed.

\section{Authorship Contributions}

Conception: H.S., J.C., H.C., A.A.Ş., G.T., M.R., B.L.; Design: H.S., J.C., H.C., A.A.Ş.; Supervision: A.A.Ş., B.L.; Data Collection or Processing: H.S., H.C., A.A.Ş., G.T.; Analysis or Interpretation: H.S., H.C., A.A.Ş., G.T.; Literature Search: H.S.; Writing: H.S., B.L.; Critical Review: H.C., A.A.Ş., G.T., B.L.

Conflict of Interest: No conflict of interest was declared by the authors.

Financial Disclosure: The authors declared that this study received no financial support.

\section{References}

1. Popescu NC, King CR, Kraus MH. Localization of the human erbB-2 gene on normal and rearranged chromosomes 17 to bands q12-21.32. Genomics 1989; 4: 362-366. (PMID: 2565881) [CrossRef]

2. Kallioniemi OP, Holli K, Visakorpi T, Koivula T, Helin HH, Isola JJ. Association of c-erbB-2 protein over-expression with high rate of cell proliferation, increased risk of visceral metastasis and poor long-term survival in breast cancer. Int J Cancer 1991; 49: 650-655. (PMID: 1682277) [CrossRef]

3. Vogel CL, Cobleigh MA, Tripathy D, Gutheil JC, Harris LN, Fehrenbacher L, et al. Efficacy and safety of trastuzumab as a single agent in first-line treatment of HER2-overexpressing metastatic breast cancer. J Clin Oncol 2002; 20: 19-726. (PMID: 11821453) [CrossRef]

4. Joensuu H, Kellokumpu-Lehtinen PL, Bono P, Alanko T, Kataja V, Asola $\mathrm{R}$, et al. Adjuvant docetaxel or vinorelbine with or without trastuzumab for breast cancer. N Engl J Med 2006; 354: 809-820. (PMID: 16495393) [CrossRef]

5. Banerjee S, Smith IE. Management of small HER2-positive breast cancers. Lancet Oncol 2010; 11: 1193-1199. (PMID: 21126688) [CrossRef]

6. Jackisch C. HER-2-positive metastatic breast cancer: optimizing trastuzumab-based therapy. Oncologist 2006; 11(Suppl 1): 34-41. (PMID: 16971738) [CrossRef]

7. Wolff AC, Hammond ME, Hicks DG, Dowsett M, McShane LM, Allison $\mathrm{KH}$, et al. Recommendations for human epidermal growth factor receptor 2 testing in breast cancer: American Society of Clinical Oncology/College of American Pathologists clinical practice guideline update. J Clin Oncol 2013; 31: 3997-4013. (PMID: 24101045) [CrossRef]

8. Tse CH, Hwang HC, Goldstein LC, Kandalaft PL, Wiley JC, Kussick SJ, et al. Determining true HER2 gene status in breast cancers with polysomy by using alternative chromosome 17 reference genes: implications for anti-HER2 targeted therapy. J Clin Oncol 2011; 29: 4168-4174. (PMID: 21947821) [CrossRef]

9. Wolff AC, Hammond ME, Schwartz JN, Hagerty KL, Allred DC, Cote RJ, et al American Society of Clinical Oncology/College of American Pathologists guideline recommendations for human epidermal growth factor receptor 2 testing in breast cancer. J Clin Oncol 2007; 25: 118145. (PMID: 17159189) [CrossRef]

10. Jang MH, Kim EJ, Kim HJ, Chung YR, Park SY. Assessment of HER2 status in invasive breast cancers with increased centromere 17 copy number. Breast Cancer Res Treat 2015; 153: 67-77. (PMID: 26223814) [CrossRef]
11. Agersborg S, Mixon C, Nguyen T, Aithal S, Sudarsanam S, Blocker F, et al. Immunohistochemistry and alternative FISH testing in breast cancer with HER2 equivocal amplification. Breast Cancer Res Treat 2018; 170: 321-328. (PMID: 29564742) [CrossRef]

12. Sneige N, Hess KR, Multani AS, Gong Y, Ibrahim NK. Prognostic significance of equivocal human epidermal growth factor receptor 2 results and clinical utility of alternative chromosome 17 genes in patients with invasive breast cancer: a cohort study. Cancer 2017; 123: 11151123. (PMID: 27893937) [CrossRef]

13. The University of Texas MD Anderson Cancer Center. Residual Cancer Burden Calculator. Available from: http://www3.mdanderson.org/app/ medcalc/index.cfm?pagename $=$ jsconvert3 [CrossRef]

14. Hammond ME, Hayes DF, Dowsett M, Allred DC, Hagerty KL, Badve $S$, et al. American Society of Clinical Oncology/College of American Pathologists guideline recommendations for immunohistochemical testing of estrogen and progesterone receptors in breast cancer. J Clin Oncol 2010; 28: 2784-2795. (PMID: 20404251) [CrossRef]

15. Press MF, Sauter G, Buyse M, Fourmanoir H, Quinaux E, Tsao-Wei DD, et al. HER2 gene amplification testing by fluorescent in situ hybridization (FISH): comparison of the ASCO-College of American Pathologists Guidelines with FISH scores used for enrollment in Breast Cancer International Research Group Clinical Trials. J Clin Oncol 2016; 34: 3518-3528. (PMID: 27573653) [CrossRef]

16. Downs-Kelly E, Yoder BJ, Stoler M, Tubbs RR, Skacel M, Grogan T, et al. The influence of polysomy 17 on HER2 gene and protein expression in adenocarcinoma of the breast: a fluorescent in situ hybridization, immunohistochemical, and isotopic mRNA in situ hybridization study. Am J Surg Pathol 2005; 29: 1221-1227. (PMID: 16096413) [CrossRef]

17. Hofmann M, Stoss O, Gaiser T, Kneitz H, Heinmöller P, Gutjahr T, et al. Central HER2 IHC and FISH analysis in a trastuzumab (Herceptin) phase II monotherapy study: assessment of test sensitivity and impact of chromosome 17 polysomy. J Clin Pathol 2008; 61: 89-94. (PMID: 17412870) [CrossRef]

18. Salido M, Tusquets I, Corominas JM, Suarez M, Espinet B, Corzo C, et al. Polysomy of chromosome 17 in breast cancer tumors showing an overexpression of ERBB2: a study of 175 cases using fluorescence in situ hybridization and immunohistochemistry. Breast Cancer Res 2005; 7: R267-R273. (PMID: 15743507) [CrossRef]

19. Takehisa M, Sasa M, Bando Y, Hirose T, Morimoto T, Nagao T, et al. Chromosomal aneusomy (chr 1, 11,17) detected by fluorescence in situ hybridization may be a prognostic factor in breast cancer. Anticancer Res 2007; 27: 1073-1078. (PMID: 17465246) [CrossRef]

20. Torrisi R, Rotmensz N, Bagnardi V, Viale G, Curto BD, Dell'orto P, et al. HER2 status in early breast cancer: relevance of cell staining patterns, gene amplification and polysomy 17. Eur J Cancer 2007; 43: 2339-2344. (PMID: 17855075) [CrossRef]

21. Vanden Bempt I, Van Loo P, Drijkoningen M, Neven P, Smeets A, Christiaens MR, et al. Polysomy 17 in breast cancer: clinicopathologic significance and impact on HER-2 testing. J Clin Oncol 2008; 26: 48694874. (PMID: 18794552) [CrossRef]

22. Varshney D, Zhou YY, Geller SA, Alsabeh R. Determination of HER2 status and chromosome 17 polysomy in breast carcinomas comparing HercepTest and PathVysion FISH assay. Am J Clin Pathol 2004; 121: 70-77. (PMID: 14750243) [CrossRef]

23. Petroni S, Addati T, Mattioli E, Caponio MA, Quero C, Rubini V, et al. Centromere 17 copy number alteration: negative prognostic factor in invasive breast cancer? Arch Pathol Lab Med 2012; 136: 993-1000. (PMID: 22938586) [CrossRef]

24. Marchiò C, Lambros MB, Gugliotta P, Di Cantogno LV, Botta C, Pasini $\mathrm{B}$, et al. Does chromosome 17 centromere copy number predict polysomy in breast cancer? A fluorescence in situ hybridization and microarray- 
based CGH analysis. J Pathol 2009; 219: 16-24. (PMID: 19670217) [CrossRef]

25. Krishnamurti U, Hammers JL, Atem FD, Storto PD, Silverman JF. Poor prognostic significance of unamplified chromosome 17 polysomy in invasive breast carcinoma. Mod Pathol 2009; 22: 1044-1048. (PMID: 19396150) [CrossRef]

26. Ma Y, Lespagnard L, Durbecq V, Paesmans M, Desmedt C, GomezGaldon M, et al. Polysomy 17 in HER-2/neu status elaboration in breast cancer: effect on daily practice. Clin Cancer Res 2005; 11: 4393-4399. (PMID: 15958623) [CrossRef]

27. Hyun CL, Lee HE, Kim KS, Kim SW, Kim JH, Choe G, et al. The effect of chromosome 17 polysomy on HER-2/neu status in breast cancer. J Clin Pathol 2008; 61: 317-321. (PMID: 17761736) [CrossRef]

28. Lal P, Salazar PA, Ladanyi M, Chen B. Impact of polysomy 17 on HER$2 /$ neu immunohistochemistry in breast carcinomas without HER-2/neu gene amplification. J Mol Diagn 2003; 5: 155-159. (PMID: 12876205) [CrossRef]

29. Downey L, Livingston RB, Koehler M, Arbushites M, Williams L, Santiago A, et al. Chromosome 17 polysomy without human epidermal growth factor receptor 2 amplification does not predict response to lapatinib plus paclitaxel compared with paclitaxel in metastatic breast cancer. Clin Cancer Res 2010; 16: 1281-1288. (PMID: 20145176) [CrossRef]

30. Wolff AC, Hammond ME, Allison KH, Harvey BE, Mangu PB, Bartlett JMS, et al. Human epidermal growth factor receptor 2 testing in breast cancer: American Society of Clinical Oncology/College of American Pathologists Clinical Practice Guideline Focused Update. J Clin Oncol 2018; 36: 2105-2122. (PMID: 29846122) [CrossRef] 